\title{
Overview of Methodologies to Sample and Assess Microbial Burden in Low Biomass Environments
}

\author{
James Bruckner and Kasthuri Venkateswaran* \\ (Biotechnology and Planetary Protection Group, Jet Propulsion Laboratory, \\ California Institute of Technology, Pasadena, CA)
}

\begin{abstract}
The detection and evaluation of microbial contamination is the goal of many programs in the medical, food, pharmaceutical, and environmental monitoring/biothreat detection industries. Similarly, NASA's Planetary Protection program strives to assess, as well as prevent, microbial contamination in spacecraft assembly facilities and on spacecraft themselves. The development of improved methods and technologies designed to facilitate these common goals is therefore important. This review will focus on instrumentation and techniques currently being utilized or evaluated for use by the Planetary Protection program in the clean room environments of spacecraft assembly facilities. Current sampling technologies (novel sampling instrumentation and different collection materials) were compared with NASA standard protocols under field and controlled conditions. A brief literature review was presented to augment the comparisons. Additionally, as the integrity of sample analyses is directly dependent on both the efficiency of sample collection and the quality of sample processing, a novel nucleic acid extraction instrument was also evaluated. The impact of these new technologies will be discussed with respect to sample collection, processing, and analyses from the low biomass environments present in NASA clean rooms, although the findings may be applied in a broader context to other industries and programs as well as to the development of future integrated biosensors.
\end{abstract}

\section{Introduction}

The mission of NASA's Planetary Protection (PP) program is similar to programs within the medical, pharmaceutical, food, and biothreat detection industries; namely the detection, identification, and prevention of microbial contamination. However, despite rigorous PP protocols and sterilization procedures, studies have indicated that spacecraft assembly facilities and the surfaces of spacecraft are not devoid of microbial life ${ }^{11,12), 28)}$. Microbial bioburden has been demonstrated to be low $\left(<10^{2}\right.$ colony forming units $(\mathrm{CFU}) / \mathrm{cm}^{2,11)}$ ), although even low levels of biomass have the potential to

\footnotetext{
* Corresponding author:

Kasthuri Venkateswaran

Biotechnology and Planetary Protection Group

M/S 89, Jet Propulsion Laboratory; California Institute of Technology

4800 Oak Grove Dr., Pasadena, CA 91109

Phone: 818-393-1481 Fax: 818-393-4176

kjvenkat@jpl.nasa.gov
}

foul pristine environments (e.g., other planetary bodies), confound future life detection missions, and possibly affect the safety and well being of crews $^{11,14), 20)}$. The development of improved sample collection, processing, and analytical instruments and techniques is therefore of special interest to NASA, as well important to both science and industry. Empirical knowledge from these instruments will lead to the integration of individual sub-systems into a biosensor to monitor microbial life.

Given that the bioburden of spacecraft and spacecraft assembly facilities has been demonstrated to be low and that the microbial communities present in these oligotrophic, desiccating environments undoubtedly have specialized growth requirements, the development of procedures and instruments designed to facilitate molecular-based analyses is paramount. While culture-based methods still yield valuable information, they are hindered by a lack of sensitivity (i.e., the inability to detect viablebut-not-cultivable organisms ${ }^{6}$ ) and are in- 
herently biased due to the use of selective media (e.g., the majority of microbes present in the environment cannot be cultured at this time $^{2)}$ ). Molecular-based methods have lower detection limits and offer a broader, more universal specificity 7$), 11,12), 15), 20), 27), 28$ making them better suited for the detection and analyses of microbial load in these environments.

This research article was not intended as a comprehensive review of current and future sample collection and sensing technologies. Rather, the information presented represents an overview of methodologies and techniques currently being utilized (or evaluated for use) to more effectively sample and assess microbial contamination in low biomass, extreme environments (i.e., spacecraft surfaces and associated assembly facilities). In additions to a brief literature review, a NASA/JPL in-house technology and swab material evaluation are discussed with respect to sample collection efficiency. This information may be applied in a broader context to address microbial detection and analyses in similar environments such as those found in the food and medical industries, as well as issues regarding the detection of biothreat agents.

\section{Materials and Methods}

\section{Sample collection and processing technology}

(i) Traditional NASA standard sampling

NASA protocols recommend the use of sterile cotton swabs for the collection of samples from spacecraft surfaces and the use of sterile polyester clean room wipes (Texwipes) when sampling larger surface areas. The standard NASA sampling protocol ${ }^{19)}$ was used when and where necessary during this study. Briefly, swabs were aseptically removed from its sterile container and the head of the swab moistened in 3 to $10.0 \mathrm{~m} l$ of sterile distilled water. The swabs were held in an angle so that the handle makes about a 30-degree angle with the surface to be sampled. The head of the swab was rotated slowly and thoroughly over a measured surface area of $25 \mathrm{~cm}^{2}$. The direction of the swabbing motion was changed to 90 degrees and again the same surface was swabbed. A third coverage of the surface to be sampled was completed by again changing the direction of the swabbing motion by 90 degrees. The head of the swab was severed below the portion of the handle touched by the operator and placed into an individual tube containing the initial moistening solution. Tubes were then vortexed at maximum power $(5-6 \mathrm{sec})$ and prior to sonication as follows: tubes were suspended in the middle of an ultrasonic bath filled to normal capacity and sonicated (frequency of $25 \mathrm{kHz}$ ) for 2 min to release microbes into the medium and the swabs removed with sterile forceps.

Areas within the Jet Propulsion Laboratory Spacecraft Assembly Facility (JPL-SAF) and the JPL Biomolecule Detection Laboratory (BDL) were sampled utilizing a protocol modified from the NASA standard procedure ${ }^{19)}$. Modifications were the use of molecular grade water instead of phosphate-buffered saline (PBS; pH 7.2) and the sampling of a larger area $\left(81 \mathrm{~cm}^{2}\right.$ vs. $\left.25 \mathrm{~cm}^{2}\right)$.

(ii) Novel sampling technology using vacuum suction sampler

A novel vacuum-based suction sampler (VSS; Lynntech, College Station, TX USA) was evaluated against current NASA standard sampling method. Samples were collected in parallel with the NASA standard swabs (see above) in accordance with VSS operating instructions. This simple to operate, field-deployable, and light weight $(<1.5 \mathrm{~kg})$ instrument (Fig. 1) consists of two sub-systems: a vacuum-based suction module and sample collection wand connected with flexible tubing. The unit uses sterile filter heads that can be used dry or premoistened and aseptically ejected into a $50-\mathrm{m} l$ tube containing collection fluid. Filter heads used to collect samples in these studies were ejected into $50-\mathrm{m} l$ tubes containing molecular grade water and processed as described previously.

\section{Microbiological examination}

(i) Traditional culture-based assays

(a) Enumeration of spores. Estimates of spore forming bacteria were obtained using the NASA standard assay ${ }^{19}$. Briefly, the samples were heat shocked $\left(80^{\circ} \mathrm{C}, 15\right.$ minutes $), 1-\mathrm{ml}$ placed in a sterile petri dish (done in duplicate), and mixed with Trypticase Soy Agar (TSA; Difco) using pour plating techniques. Pour plated samples were incubated at $32^{\circ} \mathrm{C}$ and CFU's enumerated at 24,48 , and 72 hour intervals. 


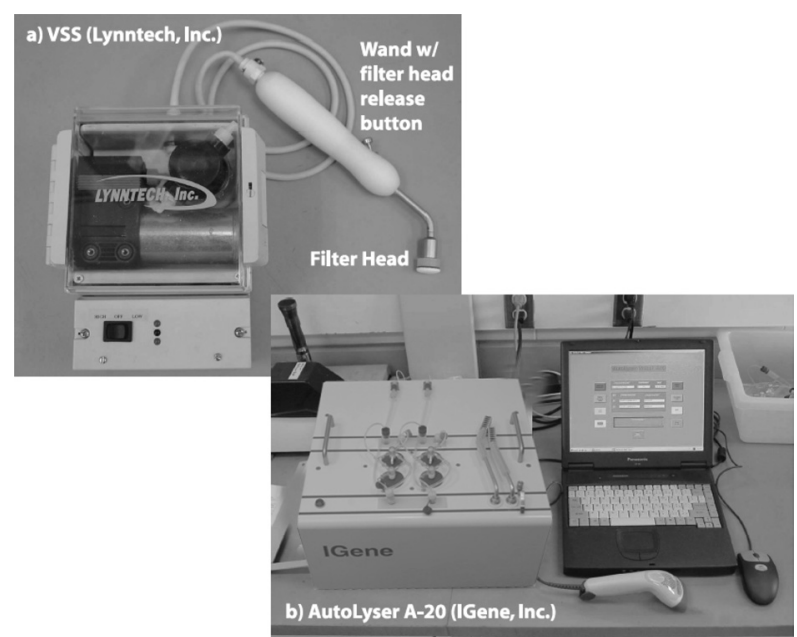

Fig. 1. Novel technology to aid in sample collection and processing include:

a) Vacuum Suction Sampler (VSS; Lynntech, Inc.)-Used to collected surface samples. Note the removable sample head and collection wand.

b) AutoLyser A-20 (IGene)-Automated nucleic acid extractor. Unit uses prepackaged sterile disposable cartridges and reagents and is controlled via laptop computer. Samples cartridges are bar coded allowing quick and error free entry into laptop's logbook.

(b) Enumeration of total cultivable bacteria. Processed but not heat shocked surface samples were plated in triplicate $(100 \mathrm{ml} /$ plate $)$ on TSA and R2A (Difco) and incubated at $32^{\circ} \mathrm{C}$ and $25^{\circ} \mathrm{C}$, respectively. Colonies were enumerated at 2-day and 7-day intervals.

(ii) Novel bio-molecules-based assays

(a) Estimation of total and viable microbial population. Total ATP (total microbes) and intracellular-ATP (viable microbes) were measured as described previously ${ }^{11,27)}$. The dynamic range of this assay is from $5 \times 10^{-12} \mathrm{M}$ to $10^{-7}$ M ATP, with one relative luminescence unit (RLU) corresponding to $2 \times 10^{-14} \mathrm{M}$ ATP as determined by linear regression analysis of standard curves with known ATP concentrations ${ }^{27}$. To verify intracellular-ATP measurements, and ensure that extracellular ATP had been fully degraded, a sterile water sample spiked with purified ATP (Sigma, St. Louis, MO) and inactivated B. subtilis cells were subjected to the ATP-eliminating procedure. No signal above background was obtained. ATP levels were measured in quadruplicate using $100 \mu \mathrm{l}$ aliquots of processed surface samples (see above). CheckLite SP and HS kits (Kikkoman Corp., San Francisco, CA) were used in accordance with manufacturer instructions to assay both total and intracellular ATP levels (measuring total bioburden and viable cells, respectively;27).

(b) Estimation of endotoxin-producing bacterial population. When microorganisms invade an animal, the immune system responds by initiating a highly specific enzyme cascade in its blood cells (amebocytes). It is known that this cascade is initiated by the presence of lipopolysaccharide (LPS) found in the outer membrane of Gram-negative bacteria and beta glucan in yeasts, and results in the formation of a gelclot that destroys the invading microbes. The LPSbased microbial detection assay exploits this principle as it occurs in amoebocytes of horseshoe crab (Limulus polyphemus) coupled with a chromogenic substrate. Hereafter, we refer to this as the limulus amebocyte assay (LAL). This method is most commonly used to quantify endotoxin and is the basis for the American Society for Testing and Materials method E2144-01. Commercially available kits (Charles River Laboratories, Wilmington, MA) were used to perform this rapid and simple assay (2-h) where appropriate aliquots of samples were mixed with reaction reagents and clotting was measured colorimetrically.

(c) Estimation of total bacterial population using quantitative-PCR. Ribosomal RNA gene copy numbers were estimated by quantitative PCR (Q-PCR). All experiments were done in triplicate using universal eubacterial primers targeting the 16S rRNA gene, 1369F (5'-CGG TGA ATA CGT TCY CGG-3') and 1492R (5'GGW TAC CTT GTT ACG ACT T-3'), and the fluorescent-labeled probe TM1389F (5'-FAMCTT GTA CAC ACC GCC CGT C-TAMRA-3 $\left.{ }^{\prime}\right)^{26)}$. Each $50 \mu l$ PCR reaction consisted of $25 \mu l$ of $2 \mathrm{X}$ Taqman Universal PCR Master Mix (Applied Biosystems Inc., Foster City, CA), $0.8 \mu \mathrm{M}$ of each oligonucleotide primer, $0.5 \mu \mathrm{M}$ of oligonucleotide probe, and $5 \mu l$ of template DNA. The remaining reaction volume was comprised of molecular grade water. Reaction conditions were as follows: $95^{\circ} \mathrm{C}$ denaturation for $15 \mathrm{~min}$, followed by 40 cycles of denaturation at $95^{\circ} \mathrm{C}$ for $15 \mathrm{~s}$ and a combined annealing and extension at $60^{\circ} \mathrm{C}$ for $1.5 \mathrm{~min}$. The Q-PCR reaction was performed in a MJ Research PTC-100 thermal cycler (Bio-Rad Lab., Waltham, MA). 


\section{(iii) DNA extraction}

Prior to DNA extraction and Q-PCR analyses, samples were concentrated by lyophilization (BenchTop 2K, VIRTIS, Gardiner, NY) and resuspended in $500 \mu \mathrm{l}$ of molecular grade water. Nucleic acid extractions were performed using an automated nucleic acid extraction instrument (AutoLyser A-20, IGene, Menlo Park, CA, USA; Fig. 1) in accordance with manufacturers instructions. Detailed protocol in using the AutoLyser is given in the following website (http://www.autolyser.com/ instrument.htm). The AutoLyser A-20 is a stand alone, fully automated, field deployable unit capable of performing nucleic acid extractions without additional manual steps (aside from initial loading). This A-20 is a closed system that utilizes disposable sample handling cartridges minimizing contact with infective or corrosive agents.

\section{Efficiency of several swab materials in remov-} ing microbes from surfaces

A laboratory study was undertaken to evaluate the performance of different swab materials (see below) using the NASA standard assay for aerobic spores ${ }^{19}$ modified for longer processing times. This assay was augmented to include Q-PCR to estimate the total number of spores collected and recovered.

(i) Spore inoculation

Bacillus subtilus var. niger spores ATCC 9372 were diluted to appropriate concentrations using sterile water and spotted directly on to sterile $25 \mathrm{~cm}^{2}$ aluminum 6061 coupons. Initial CFU titers (enumeration on TSA after incubated at $32^{\circ} \mathrm{C}$ for 24,48 , and 72 hours) were performed at the time of inoculation to ensure accurate spore concentrations. Coupons were inoculated in quintuplicate at each concentration to account for the three materials to be tested, a swab blank, and a water control. Five replicate series were performed per experiment. Two separate experimental runs were performed with concentrations of $10^{4}, 10^{3}$, and 0 (blank) CFU/coupon and $10^{3}, 10^{2}$, and 0 (blank) $\mathrm{CFU} /$ coupon.

\section{(ii) Swabs}

The swabs utilized for the experiments were autoclave sterilized prior to use. The swab materials used were cotton (Sterile Cotton Applicators, no. 25-806-1WC, Puritan, Guilford, ME USA), polyester (CleanTips ${ }^{\circledR}$ Swabs, no.
TX761, Texwipe, NJ USA), and macrofoam (CleanTips ${ }^{\circledR}$ Swabs, no. TX707A, Texwipe, NJ USA).

(iii) Sample collection and processing

Coupons were swabbed in accordance with the NASA standard assay as described previously. Sterile swabs were pre-wetted with sterile water and coupons sampled by swabbing horizontal S-patterns. The coupon was then rotated $90^{\circ}$ and sampled in the same fashion. Swabs were then placed into screw cap tubes containing $5-\mathrm{m} l$ of sterile phosphate buffer (PBS). Tubes containing swabs were subjected to 5 minutes vortexing, followed by 3 minutes of sonication, and another 5 minutes of vortexing. Samples were then aliquoted as follows: 2$\mathrm{m} l$ for culture based assay and $3-\mathrm{m} l$ for DNA extraction and subsequent Q-PCR. For both experiments, the following controls were used: a coupon blank (0 CFU/coupon) to evaluate coupon sterility and a swab blank (designated sterile water, SW) to evaluate swab sterility.

(iv) DNA extraction and estimation of total spores

Nucleic acids were extracted from 3-m $l$ aliquots using a protocol ${ }^{24)}$ modified to include a series of freeze/thaw cycles. Pellets were resuspended in $50-100 \mu \mathrm{l}$ of TE-buffer $(100 \mathrm{mM}$ Tris-HCl, 10 mM EDTA; pH 8.0) overnight at room temperature and stored at $-80^{\circ} \mathrm{C}$. Q-PCR was performed as previously described.

\section{Results and Discussion}

The purpose of this review was to briefly summarize the current state of the technology and methods being utilized and evaluated for the collection and analyses of 'total' microorganisms from spacecraft and spacecraft assembly facilities. JPL in-house laboratory studies undertaken to evaluate a novel mechanical surface sampler (VSS) as well as several different types of swab materials (cotton, polyester, and macrofoam) have been included. Direct comparisons to the NASA standard assay (a culturebased method; ${ }^{19)}$ ) were used in parallel with modern molecular techniques to assess performance. As the limitations of culture based methods are widely accepted ${ }^{7)}$ the focus will primarily be on the collection of microbial samples for molecular analyses. Previous studies using molecular methods, namely an ATP 


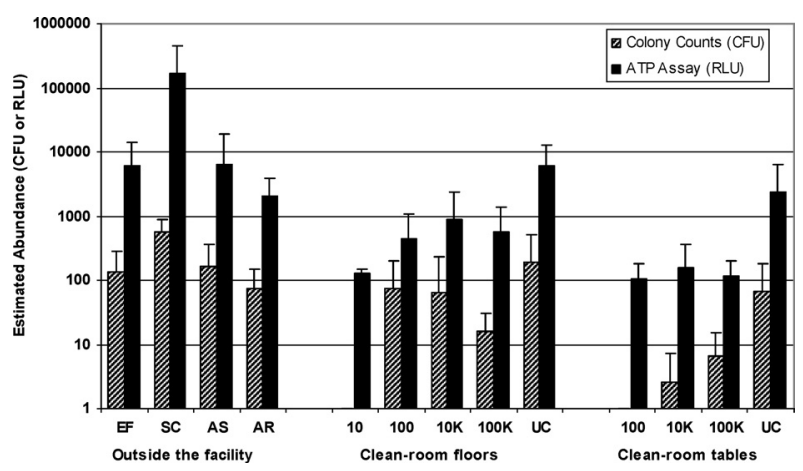

Fig. 2. Microbial contamination in spacecraft assembly facilities as demonstrated by a culturedependent and a molecular-based (ATP) assay [ref]. The ATP assay indicates a 1 to $3 \log$ higher bioburden than the tradition culturebased methods. Abbreviations are as follows: EF-entry floor, SC-shoe cleaner, AS-air shower, AR-anteroom floor. Clean rooms are designated by the particles limits as 10, 100, $10 \mathrm{~K}(10,000), 100 \mathrm{~K}(100,000)$, and UC (unclassified).

assay $^{27)}$ and LAL assay), have clearly illustrated the advantages of these techniques over culture-based analyses in clean-room like environments.

A comparison of 650 samples taken from clean room floors, tables, benches, and other locations ${ }^{27)}$ demonstrated that when the viable microbial community was evaluated using an ATP assay, estimates were one to 3-logs higher than indicated by aerobic plate counts (Fig. 2). Although both the cultivable and ATP-based determinations indicated that the microbial burden was lower in clean-room facilities than in ordinary rooms (Fig. 2), no direct correlation could be drawn between the two sets of measurements because each assay measured a different microbial community subset. A large fraction of the samples yielded no colony formers on TSA, but were positive for viable microorganisms based on intracellular ATP content. Subsequently, genomic DNA was isolated directly from selected samples and $16 \mathrm{~S}$ rDNA fragments were cloned and sequenced, identifying nearest neighbors, many of which were known to be noncultivable in the media employed $^{27)}$. It was concluded that viable microbial contamination can be reliably monitored by measurement of intracellular ATP, and that this method may be considered superior to cultivable colony counts due to its speed and its ability to report the presence of viable but non-

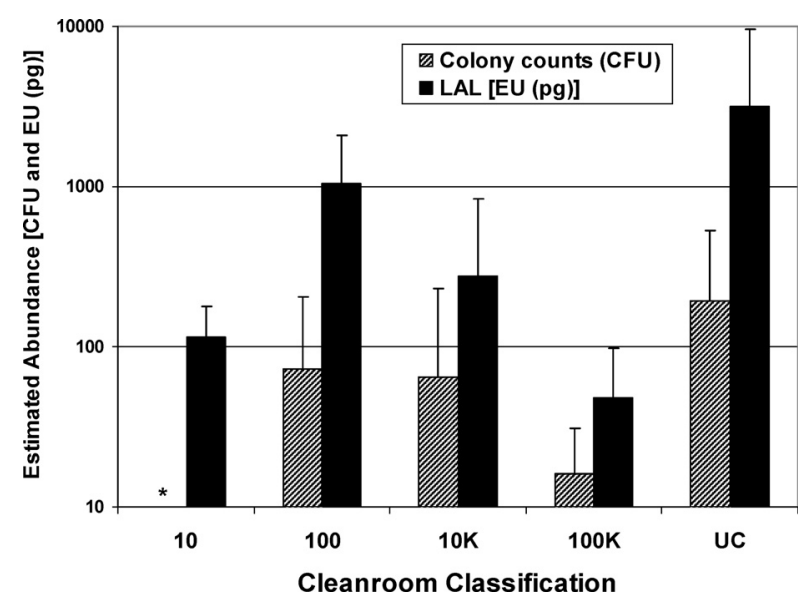

Fig. 3. Microbial contamination of spacecraft assembly room floors as determined by culture- and a molecular-based (LPS detection) assays [ref]. A 1 to 2 log increase in bioburden was detected using the LPS-based Limulus amoebocyte assay (LAL) when compared with tradition culture-based analyses. Clean room classifications are as presented in Figure KJV1.

* Note that no colonies were detected in the class 10 cleanroom.

cultivable organisms. When the detection of nonviable microbes is of interest, the ATP assay can be supplemented with DNA analysis.

Similarly, the LAL assay was chosen to measure the microbial contamination of various JPL clean rooms ${ }^{5}$. Uncertified areas such as entrance floors, shoe cleaners, air-showers, and ante-rooms contained quantifiable levels of contamination (equivalent to $10^{2}$ to $10^{3} \mathrm{pg} / \mathrm{cm}^{2}$ endotoxin), whereas, most of the certified clean room facilities did not. LAL assay-based microbial burden was observed to be equivalent to or only one-log higher (for shoe-cleaner areas) than measured by cultivable counts (Fig. 3). The low microbial burden seen in this study (compared with estimates using the ATP assay) could be explained by the inherent limitation of the LAL assay; namely that the detected endotoxin molecule is not produced by the Grampositive bacteria which dominated the cultivable counts. The combination of the conventional microbial assay, which is laborious and timeconsuming $(\sim 72 \mathrm{~h})$ and the rapid LAL assay $(\sim 1 \mathrm{~h})$ was necessary to assess the total gross microbial contamination.

Microscopy was not practical for use in assessing these surfaces because of the drastically low numbers of microorganisms (0 to $10^{2}$ CFU per $25 \mathrm{~cm}^{2}$ ) present. 


\section{Surface sampling}

Procedures and technologies designed to collect microbial samples from surfaces were well documented in the literature, although numerous of the technologies were tailored exclusively for the culture-based collection of target organism (i.e., coliforms, specific pathogens, etc.). As previously mentioned, these media-based technologies will not be discussed in this review. Published reports of non-media based technologies involving the use of swabs, wipes, sponges, and vacuumbased samplers $\left.\left.\left.\left.{ }^{3)}, 4\right), 8,10\right), 17\right), 18\right), 22,23$ ) were compared with respect to the following properties: material used (cotton, polyester, rayon, macrofoam), area of sample collected (large vs. small), and sampling conditions (wet vs. dry). These reviews were supplemented with data obtained from NASA/JPL evaluations.

Published data indicated that macrofoam was a distinctly better material with respect to both collection and recovery efficiency than were polyester or rayon. Macrofoam swabs were demonstrated to collect $30 \%$ more spores $^{8), 22)}$ even at spore concentrations $<10^{1}$ spores per preparation ${ }^{8)}$. Recovery efficiencies $>90 \%$ were achieved from macrofoam swabs during direct inoculation studies (recovery efficiency from rayon and polyester was decidedly lower ${ }^{22)}$ ). Electron micrographs of various swab materials show the more uniform distribution of pores/collection areas within macrofoam, which may be the reason for the materials superior performance.

A NASA/JPL swab material study evaluating cotton, polyester and macrofoam swabs supported these published studies. Using the culture-based NASA standard assay ${ }^{19)}$ and molecular-based Q-PCR, the three swab materials were evaluated for collection and recovery efficiency of Bacillus subtilus var. niger spores plated on aluminum coupons. Based on CFU enumeration, cotton and macrofoam swabs outperformed polyester swabs by an order of magnitude (Fig. 4). Although estimates of 16S copy number using Q-PCR indicated similar collection and recovery efficiency for all three materials (Fig. 5), cotton swabs showed higher levels of contamination (by an order of magnitude) in the blank. Based on the results of the NASA standard assay (cotton $>$ macrofoam $\gg \gg$ poly-

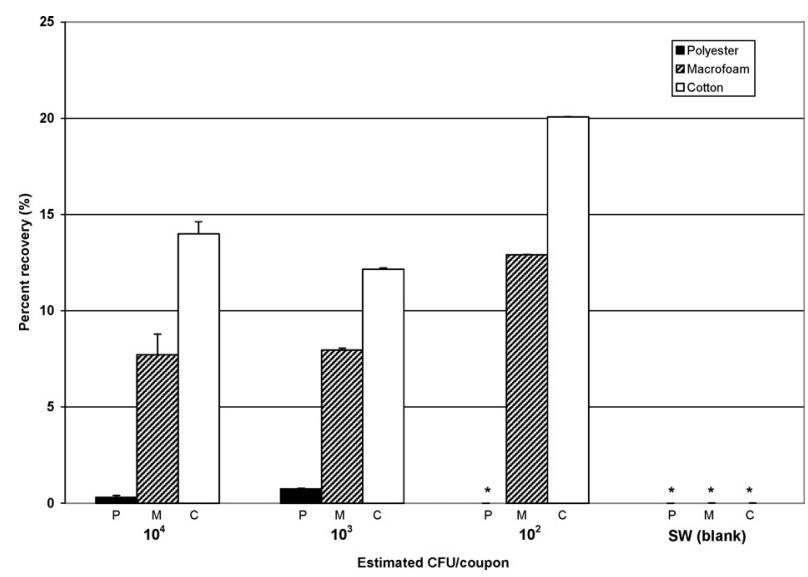

Fig. 4. Swab material analyses using culture-based method. Results are expressed as percent recovery of the initial spore inocula on an aluminum (6061) coupon. As the results from the coupon blank and swab blank (SW) were identical (no CFUs were detected), only SW is presented. Asterisks are placed on the chart to represent zero values.

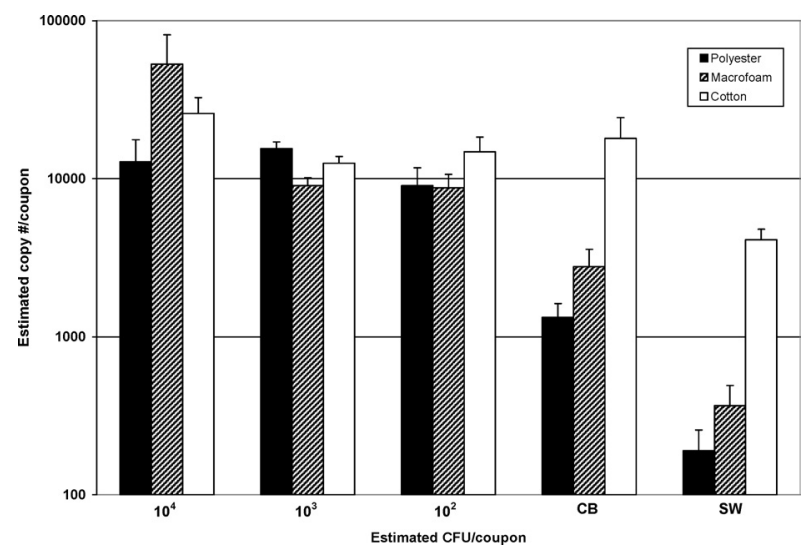

Fig. 5. Swab material analyses using molecular method (Q-PCR). Results are expressed as estimated copy $16 \mathrm{~S}$ copy number per coupon (estimates back calculated based on Q-PCR results). Note the elevated copy numbers with respect to cotton in both the coupon blank (CB) and the swab blank (SW).

ester) and Q-PCR (macrofoam $>$ polyester $\gg$ cotton), swabs composed of macrofoam performed the best overall with respect to the collection of spores from coupons.

The use of mechanical aids during sampling (e.g., vacuum/suction sampling) was not shown to significantly increase collection efficiency when compared to polyester

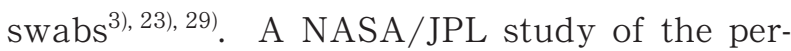
formance of the VSS against polyester swabs (Fig. 6) demonstrated the two techniques to have similar efficiencies based on both culturebased and molecular methods ${ }^{3)}$ (Figs. 7, 8). Although macrofoam swabs were not directly 


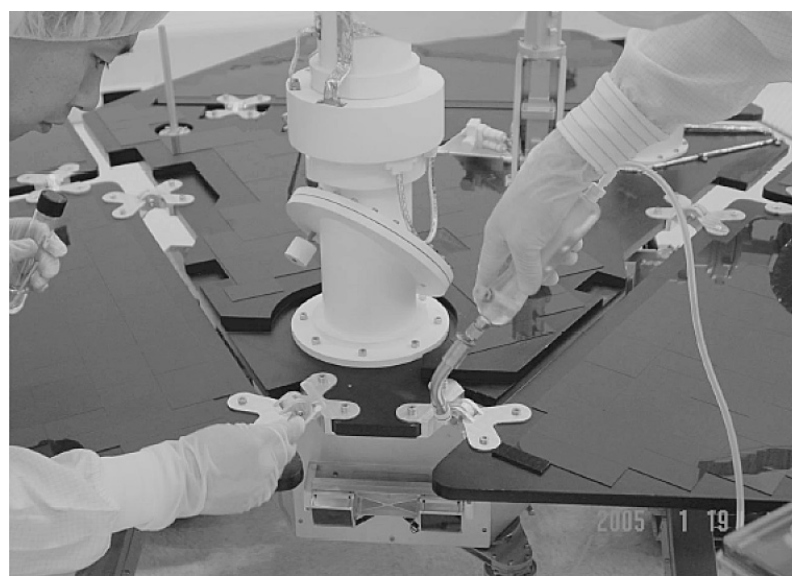

Fig. 6. Sampling the forward hinge plates on the Mars Exploratory Rover (MER) mock-up located in the JPL spacecraft assembly facility. The NASA standard swab is pictured on the left and the VSS (Lynntech, Inc.) in on the right. Samples (swab or filter heads) were placed in tubes containing molecular grade water (a tube containing a swab in water can be seen on the left side of the figure).

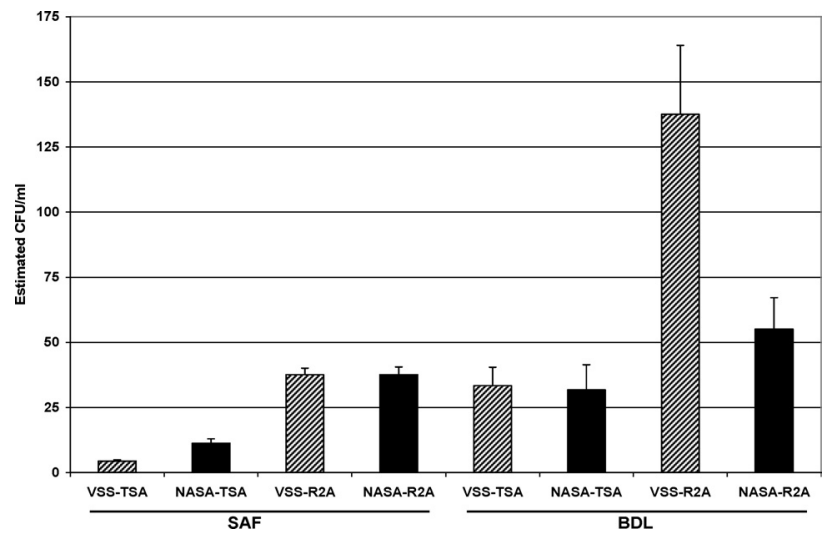

Fig. 7. Culture-based plate count data comparing a vacuum sampler (VSS) with NASA standard polyester swabs. Samples were collected and analyzed from two different JPL clean rooms: the spacecraft assembly facility (SAF) and the biomolecule detection laboratory (BDL). Trypticase soy agar (TSA) and R2A hetertrophic media were used as growth media. Results indicate that the two collection methodologies perform similarly with respect to the collection of viable cells.

tested against vacuum or suction samplers, it can be inferred from previous material tests ${ }^{8), 22)}$ that swabs composed of macrofoam would outperform the mechanical samplers. It would be interesting to evaluate a mechanical sampler with a macrofoam based collection apparatus, however, no such instrument current exists.

Studies of sample area have clearly demonstrated that the larger the area sampled in low biomass environments, the more biomass

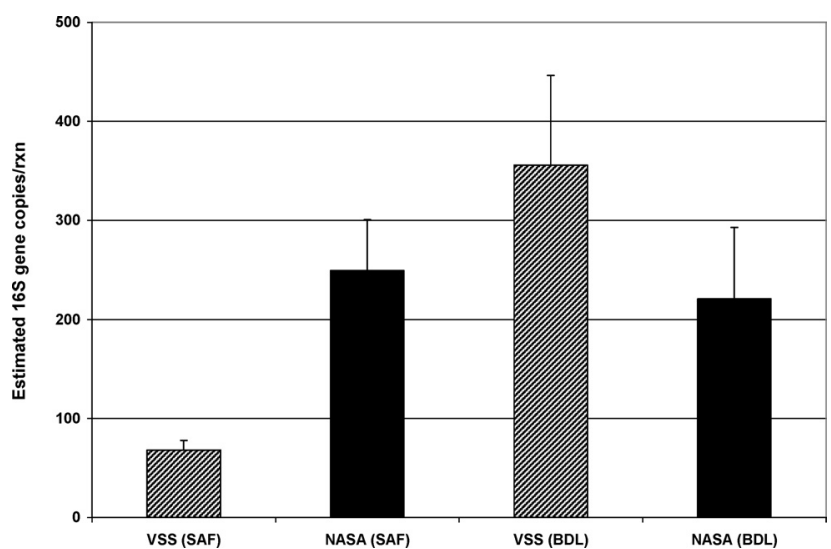

Fig. 8. Estimates of $16 \mathrm{~S}$ gene copy numbers in samples collected using the VSS and NASA standard polyester swabs. Samples were collected from the JPL-SAF and the BDL and copy number determined using Q-PCR. The data indicate both methods to have similar efficiencies.

collected $^{4), 10)}$. The effect of material used was lessened when the sample area was increased (e.g., when sampling areas of $1 \mathrm{~m}^{2}$, polyester wipes and macrofoam sponges displayed similar collection efficiencies per unit area and collected more biomaterial than the smaller swabs $^{4), 10)}$ ). It can be reasoned that the collection of more biomass, particular from low biomass environments, will result in a more complete genomic inventory (e.g., list of contaminants) of said environment.

Regardless of the material used, premoistened samplers always demonstrated higher collection efficiencies than dry samplers $^{3), 8), 17), 22), 23)}$. On average, wet-collection efficiency was shown to be $10-30 \%$ higher than dry-collection, although it should be noted that Buttner et al. $\left.{ }^{4}\right)$ indicated that spore recovery from wet material was decreased due to spores trapped in the wet samplers. This loss of recovery efficiency was estimated to be $5^{-}$ $10 \%$, and was offset by the significantly higher collection efficiency.

Based on the surveyed literature and inhouse studies, the best method for the detection of microbial contamination of clean room surfaces is to use a macrofoam-based collection apparatus that have been pre-moistened. Additionally (as well as obviously), the larger the sample collection area, the more biomass will be collected resulting in better samples.

\section{Aerosol sampling}

Although numerous types of air samplers 
are available, most are not suited to use in spacecraft assembly facilities. Impaction and gravity sampling technologies are well characterized with respect to culture-based methods, however, these technologies do not lend themselves to molecular characterization. Electrostatic deposition, while initially promising, is fairly untested at this stage in its development and available published data very limited. Additionally, the use of high voltage equipment near spacecraft is fraught with potential problems. The remaining aerosol collection technologies (filtration and impingement) represent the only viable options for aerosol sampling in these facilities.

Filtration samplers collect samples by means of the passage of airflow through, and collection of airborne particles onto a porous material. Impingement technology utilizes the forced deposition of particles into a liquid substrate, most often a buffer that can sustain its biomolecular load for future culture, microscopic, and molecular methods.

A comparison of the two methods indicated that collection efficiencies for filtration systems are generally greater than that of impingement devices, especially with respect to particles $>0.2 \mu \mathrm{m}^{1), 16), 25)}$. Filtrations systems with appropriate filters have been shown to remove $>98 \%$ of $>0.3 \mu \mathrm{m}$ particles and collect $74 \%$ of airborne Gram negative bacteria (1), whereas impingement technology collected $50-90 \%$ of $>0.3 \mu \mathrm{m}$ particles ${ }^{1,9)}$ ) and demonstrate a similar decrease in the number of Gram negative bacteria collected. Additionally, filtration systems were demonstrated to be capable of collecting significantly larger volume samples (up to 2 orders of magnitude) than impingement technolog $y^{16), 21), 25)}$. Given the presumed low biomass of spacecraft assembly facilities, the volume of air which can be sampled and the efficiency of sampling make filtration technology the best currently available option for the microbial detection in spacecraft assembly facilities and other clean-rooms.

\section{Sample processing technology}

Given that these environments contained low biomass, it was important to develop procedures and instruments to optimize sample processing efficiency. Surface and air samples were collected from the spacecraft assembly facilities of various NASA centers using both standard NASA protocols and the previously discussed VSS. All samples were processed using the AutoLyser A-20 automated nucleic acid extractor (IGene, Menlo Park, CA). The efficiency of nucleic acid extraction was evaluated by a microbial burden assay based on determination of $16 \mathrm{~S}$ rDNA copy number using Q-PCR. Use of the automated sample processor allowed extraction of nucleic acids from low biomass samples $\left(<10^{4} \mathrm{cells} / \mathrm{m} l\right)$, as well as from cultures of known International Space Station and spacecraft assembly facility contaminants, including Burkholderia cepacia, Stenotropomonas maltophilia, and Bacillus pumilis spores. Comparisons of nucleic acids extraction efficiencies indicated that the Autolyser, performed either equally as well ${ }^{3)}$ or better ${ }^{13)}$ than manual phenol:chloroform extractions. Further studies will be performed to assess whether the AutoLyser has a universal efficiency (e.g., extracts nucleic acids from all microbes) or if there are any inherent biases.

\section{Future}

Advances in sample collection technology and molecular analytic methods allow for more precise and accurate detection of microbial contamination. Given that clean room environments contained low biomass, it was important to develop and evaluate procedures and instruments designed to optimize sample collection and processing efficiency. Instrumentation such as the VSS and AutoLyser have demonstrated that novel technologies have efficiencies comparable to, if not greater than the current NASA protocols. Although these instruments decrease the time and effort required to collect and process samples, it is important to note that they are still separate, multi-step processes. The future development of integrated biosensors may combine the technologies and/or methods presented here, marrying sample collection, processing and microbial detection into a single seamless process. This integration will benefit all programs concerned with the detection and identification of microbial communities (i.e., NASA's PP program, food safety, biothreat detection) by increasing the efficiency of sample collection and processing and decreasing the time required. 


\section{Acknowledgements}

This research was carried out at the California Institute of Technology's Jet Propulsion Laboratory under a contract with NASA. This research was funded by the Advanced Environmental Monitory and Control, NRA ROSS 2005, and Mars Program Office. We thank M. Flusche, Lynntech for the VSS sampling system and R. Fields, IGene Inc. for the Autolyser system optimization and validation. We are grateful to N. Hattori and A. Matsuyama, Kikkoman Corp. for ATP measurements, S. Chung for LAL measurements, and M. Satomi for SAF sampling. We acknowledge members of Biotechnology and Planetary Protection Group for several technical help. We are grateful to Dr. H. Nakanishi for critically going through the manuscript.

\section{References}

1) Agranovski, I. E., Agranovski, V., Grinshpun, S. A., Reponen, T. and Willeke, K.: Collection of airborne microorganisms into liquid by bubbling through porous medium. Aerosol. Sci. Tech., 36, 502-509 (2002).

2) Amann, R. I., Ludwig, W. and Schleifer, K. H.: Phylogenetic identification and in situ detection of individual microbial cells without cultivation. Microbiol. Rev., 59, 143-169 (1995).

3) Bruckner, J. C., Yalamanchili, R. C., Fields, R., Sumner, R. and Venkateswaran, K.: Systematic sampling and processing of nucleic acids for low biomass samples. Presented at the 105th General Meeting of the American Society of Microbiology, Atlanta, GA (2005).

4) Buttner, M. P., Cruz, P., Stetzenbach, L. D., KlimaComba, A. K., Stevens, V. L. and Emanuel, P. A.: Evaluation of the Biological Sampling Kit (BiSKit) for large-area surface sampling. Appl. Environ. Microbiol., 70, 7040-7045 (2004).

5) Chung, S., Venkateswaran, K., Echeverria, C., La Duc, M., Schubert, W., Kazarians, G., Wainwright, N., Basic, C. and Kern, R.: Microbial populations in spacecraft assembly facilities as determined by LAL assay. Presented at the 101st General Meeting of the American Society for Microbiology, Orlando, FL (2001).

6) Grimes, D. J., Mills, A. L. and Nealson, K. H.: The importance of viable but nonculturable bacteria in biogeochemistry, p. 209-227. In Colwell, R. R. and Grimes, D. J. (eds.), Nonculturable microorganisms in the environment. ASM Press, Washington, D.C. (2000).

7) Guarnieri, V., Gaia, E., Battocchio, L., Pitzurra, M., Savino, A., Pasquarella, C., Vago, T. and Cotronei, V.: New methods for microbial contamination monitoring: an experiment on board the MIR orbital station.
Acta Astronaut., 40, 195-201 (1997).

8) Hodges, L. R., Rose, L. J., Peterson, A., Noble-Wang, J. and Arduino, M. J.: Evaluation of a macrofoam swab protocol for the recovery of Bacillus anthracis spores from a steel surface. Appl. Environ. Microbiol., 72, 4429-4430 (2006).

9) Juozaitis, A., Willeke, K., Grinshpun, S. A. and Donnelly, J.: Impaction onto a glass slide or agar versus impingement into a liquid for the collection and recovery of airborne microorganisms. Appl. Environ. Microbiol., 60, 861-870 (1994).

10) Kirschner, L. E. and Puleo, J. R.: Wipe-rinse technique for quantitating microbial contamination on large surfaces. Appl. Environ. Microbiol., 38, 466-470 (1979).

11) La Duc, M. T., Kern, R. and Venkateswaran, K.: Microbial monitoring of spacecraft and associated environments. Microbial. Ecol., 47, 150-158 (2004).

12) La Duc, M. T., Nicholson, W., Kern, R. and Venkateswaran, K.: Microbial characterization of the Mars Odyssey spacecraft and its encapsulation facility. Environ. Microbiol., 5, 977-985 (2003).

13) La Duc, M. T., Osman, S., Bruckner, J. and Venkateswaran, K.: Evaluation of methods for purification of biomolecules from low-biomass samples: Assessment of overall yield and conservation of microbial diversity. Q-236. Presented at the 107th General Meeting of the American Society for Microbiology, Toronto, Canada (2007).

14) La Duc, M. T., Pierson, D. L. and Venkateswaran, K.: Evidence of pathogenic microbes in the International Space Station drinking water: Reason for concern? Habitation, 10, 39-48 (2004).

15) Makimura, K., Hanazawa, R., Takatori, K., Tamura, Y., Fujisaki, R., Nishiyama, Y., Abe, S., Uchida, K., Kawamura, Y., Ezaki, T. and Yamaguchi, H.: Fungal flora on board the Mir-Space Station, identification by morphological features and ribosomal DNA sequences. Microbiol. Immunol., 45, 357-363 (2001).

16) Mehta, S. K., Bell-Robinson, D. M., Groves, T. O., Stetzenbach, L. D. and Pierson, D. L.: Evaluation of portable air samplers for monitoring airborne culturable bacteria. Am. Ind. Hyg. Assoc. J., 61, 850-854 (2000).

17) Moore, G. and Griffith, C.: A comparison of surface sampling methods for detecting coliforms on food contact surfaces. Food. Microbiol., 19, 65-73 (2002).

18) Moore, G. and Griffith, C.: A comparison of traditional and recently developed methods for monitoring surface hygiene within the food industry: an industry trial. Int. J. Environ. Health Res., 12, 317-329 (2002).

19) NASA: 1980. Standard Procedures for the Microbiological Examination of Space Hardware, NHB 5340.1 B, Rev. B. National Aeronautics and Space Administration, Washington, D.C.

20) Pierson, D. L.: Microbial contamination of spacecraft. Gravitational and Space Biology Bulletin, 14, 1-6 (2001).

21) Radosevich, J. L., Wilson, W. J., Shinn, J. H., DeSantis, T. Z. and Andersen, G. L.: Development of a high- 
volume aerosol collection system for the identification of air-borne micro-organisms. Lett. Appl. Microbiol., 34, 162-1677 (2002).

22) Rose, L., Jensen, B., Peterson, A., Banerjee, S. N. and Srduino, M. J.: Swab materials and Bacillus anthracis spore recovery from nonporous surfaces. Emerg. Infect. Dis., 10, 1023-1029 (2004).

23) Sanderson, W. T., Hein, M. J., Taylor, L., Curwin, B. D., Kinnes, G. M., Seitz, T. A., Popovic, T., Holmes, H. T., Kellum, M. E., McAllister, S. K., Whaley, D. N., Tupin, E. A., Walker, T., Freed, J. A., Small, D. S., Klusaritz, B. and Bridges, J. H.: Surface sampling methods for $\mathrm{Ba}$ cillus anthracis spore contamination. Emerg. Infect. Dis., 8, 1145-1151 (2002).

24) Schmidt, T. M., DeLong, E. F. and Pace, N. R.: Analysis of a marine picoplankton community by $16 \mathrm{~S}$ rRNA gene cloning and sequencing. J. Bacteriol., 173, 43714378 (1991).

25) Sigaev, G. I., Tolchinsky, A. D., Sigaev, V., Soloviev, I., K. G., Varfolomeev, A. N. and Chen, B. T.: Develop- ment of a cyclone-based aerosol sampler with recirculating liquid film: Theory and experiment. Aerosol. Sci. Tech., 40, 293-308 (2006).

26) Suzuki, M. T., Taylor, L. T. and DeLong, E. F.: Quantitative analysis of small-subunit rRNA genes in mixed microbial populations via $5^{\prime}$-nuclease assays. Appl. Environ. Microbiol., 66, 4605-4614 (2000).

27) Venkateswaran, K., Hattori, N., La Duc, M. T. and Kern, R.: ATP as a biomarker of viable microorganisms in clean-room facilities. J. Microbiol. Meth., 52, 367-377 (2003).

28) Venkateswaran, K., Satomi, M., Chung, S., Kern, R., Koukol, R., Basic, C. and White, D. C.: Molecular microbial diversity of a spacecraft assembly facility. Syst. Appl. Microbiol., 24, 311-320 (2001).

29) Whitfield, W. J., Beakley, J. W., Dugan, V. L., Hughes, L. W., Morris, M. E. and McDade, J. J.: Vacuum probe: new approach to the microbiological sampling of surfaces. Appl. Microbiol., 17, 164-168 (1969). 